\title{
EL COLEGIO: PRIMER ESCENARIO PARA APRENDER DE SEGUROS
}

\author{
The school: the first scenario to learn about insurance
}

Mónica Patricia Novoa Ávila monicanovoa1017@gmail.com Hugo Armando Guzmán Useche haguzmanu@poligran.edu.co

Institución Universitaria Politécnico Grancolombiano Especialización en Gerencia de Riesgos y Seguros Colombia

\begin{abstract}
Resumen
El presente reporte de investigación se basa en el proyecto bautizado “Insólito", que fue diseñado y liderado por la Fundación Junior Achievement Colombia (FJAC). El proyecto invita a los jóvenes a conocer, identificar y reflexionar sobre los seguros, los cuales proveen protección y respaldo para las pérdidas imprevistas, evitando el retraso y abandono de los deseos y sueños propios de cada individuo; lo cual, indica la experiencia, es lo que suele suceder cuando ocurren imprevistos y se ven forzados a luchar "exclusivamente" por subsistir durante la etapa más productiva de la vida. Por medio de juegos y actividades lúdicas se pretende involucrar al joven en el mundo de los seguros.

Los jóvenes que participaron en el proyecto se encontraban cursando décimo grado y se buscaba que se informaran y tomaran buenas decisiones en la adquisición de productos de seguros. Para este grupo se acercaba el momento de salir a la fuerza de trabajo, de independizarse, de consumir productos y servicios, de manera independiente a su núcleo familiar.

La respuesta de los jóvenes fue satisfactoria, no se encontraban alejados de los beneficios que genera el comprar una póliza, fueron conscientes que existe la necesidad de hacerlo y les llamó la atención la información suministrada.
\end{abstract}

Palabras clave: Seguros, Educación Financiera, Servicios financieros, Educación en seguros. 
MÓNICA NOVOA, HUGO GUZMÁN

\begin{abstract}
This research report is based on the project called "Insólito", which was designed and led by the Fundación Junior Achievement Colombia (FJAC). The project invites young people to know, identify and reflect on insurance, which provides protection and support for unforeseen losses, avoiding the delay and abandonment of the desires and dreams of each individual; which, experience indicates, is what usually happens when unforeseen events occur and they are forced to fight "exclusively" to survive during the most productive stage of life. Through games and recreational activities it is intended to involve young people in the world of insurance.

The young people who participated in the project were in the tenth grade and it was sought that they were informed and made good decisions in the purchase of insurance products. For this group, the time was approaching to go out into the work force, to become independent, to consume products and services, independently of their family nucleus.

The response of the young people was satisfactory, they were not far from the benefits generated by buying a policy, they were aware that there is a need to do so and the information provided caught their attention.
\end{abstract}

Key Words: Insurance, Financial education, Financial services, Insurance education.

$\begin{array}{ll}\text { Recepción: } & 17.03 .2021 \\ \text { Aceptación: } & 12.05 .2021\end{array}$

Cite este artículo como: Novoa-Ávila, M. (2021). El colegio: primer escenario para aprender de seguros. Working papers FNGS, 2(2), pp. 24-38. 
MÓNICA NOVOA, HUGO GUZMÁN

\section{INTRODUCCIÓN}

\section{La importancia de la Educación Financiera}

La educación financiera, para algunos un concepto desconocido y para otros sin importancia, se considera como la capacidad con que cuentan los individuos para agrupar, analizar, gestionar y evaluar toda clase de información relacionada con los títulos valores (dinero, propiedades, títulos al portador, inversiones a corto y mediano plazo), esto con el fin de garantizar que sus decisiones sean lo más acertadas posible (Marriot y Mellett, 1996).

Cuando hay total ausencia de este conocimiento, es cuando se ve la necesidad de crear y desarrollar programas de Educación Financiera, que puedan ir enfocados a personas agrupadas por estratos, nivel de educación, actividad económica, intereses en inversión y afines. Esta necesidad también se puede observar desde otra óptica, la de las entidades financieras y aseguradoras que necesitan explicar sus productos, en qué consisten, cómo operan, cuáles beneficios ofrecen; para que esto sea entendido, los consumidores deben conocer y entender, así sea de manera general, los conceptos básicos.

La competencia globalizada hace que los productos financieros sean cada vez más complejos, lo que hace que no todos los consumidores tengan acceso a la información suficiente para tomar la decisión de comprarlos o no. Hay evidencia de casos en los cuales una mala asesoría puede redundar en pérdida de dinero, o que se adquieran productos que no cubren las necesidades en su totalidad (Ansah y Kissi 2013).

Durante los años cincuenta y hasta los ochenta, en 29 Estados de los Estados Unidos se tuvo la iniciativa de crear políticas que fomentaran la educación financiera en estudiantes de secundaria. Esta política estuvo en auge a comienzos del siglo XXI (Agha, 2002) y (Barber, 2004). El primer tema que se tuvo en cuenta en ese país fue el ahorro, era preocupante que, aunque el gobierno tenía incentivos para que los ahorradores se multiplicaran, las estadísticas reportaban todo lo contrario. No se había creado esta cultura y no se evidenciaba, por parte de los estadounidenses, el reconocimiento de los beneficios de ahorrar. No se sentían vulnerables y creían que todo estaba bajo control. Tiempo después, las empresas se encontraron con la problemática que sus empleados no tenían un programa de ahorro para su vejez y retiro; para esto, los empleadores se pusieron en la tarea de diseñar un programa de educación enfocada en las pensiones, con el ánimo de poder ofrecer información a los empleados que los incentivara a pensar en su vejez y empezar a ahorrar e invertir para garantizar su bienestar.

En el Reino Unido se vivió algo parecido, las personas económicamente activas no entendían las diferentes opciones para pensionarse que ofrecía su Gobierno, la población inglesa desconfiaba del sistema de pensiones. La falta de claridad y conocimiento en el sistema, cuáles eran sus derechos y cuáles sus deberes, los requisitos para la jubilación hacían que la incertidumbre reinara y no se decidieran a ser beneficiarios de este programa. Para contrarrestar lo que estaba ocurriendo, el gobierno optó por crear un programa de educación financiera en pensiones, que permitiera a la población tener acceso a la información y a explicaciones en términos claros, al mismo tiempo se incluyó información sobre los planes de retiro que se ofrecían para así poder aumentar los beneficiarios de estos (Figueroa, y otros s.f.)

Un análisis en el comportamiento individual y colectivo de los individuos que han tenido acceso a los planes de educación financiera reveló que a través de la historia los planes que se han realizado con esfuerzos tanto del gobierno como de las empresas privadas, han generado una gran incidencia en las decisiones que se toman (Banco de Desarrollo de América Latina 2013); los resultados fueron contundentes, por ejemplo, la educación sobre planes de retiro en Estados Unidos tuvo una influencia positiva en el comportamiento financiero de los hogares, se incrementó el ahorro en general, como el pensional. 
Las personas que tuvieron acceso a la educación financiera, en edades tempranas, tenían mayor intención de ahorro y acumulación de activos (Bernhaim, 1997) y (Welch, 1982).

Algo que han dejado a la luz estos últimos años, y de acuerdo con el estudio realizado por CEMLA en el último trimestre de 2016; es que toman mejores decisiones las personas que cuentan con conocimientos financieros, sobre presupuestos, créditos, opciones de pago, así como los beneficios que otorgan las entidades financieras por pronto pago, los alivios financieros que hay en el mercado y cómo se puede acceder a ellos (Lusardi y Mitchell 2016).

La educación básica sobre planes de retiro influyó positivamente en el comportamiento financiero de los hogares, tanto en el ahorro, en etapa productiva, como en la vejez. Lo que buscaban era generar impacto positivo en las decisiones financieras que tomaran las personas que tuvieron acceso al programa. (Madrian y Shea, 2001).

En el 2004 realizaron un estudio sobre el programa financiero IDA en Estados Unidos, este programa se encontraba dirigido a personas de niveles de ingreso bajo; el resultado que lograron los investigadores con estudios estadísticos y matemáticos fue que se veía un aumento en el ahorro de estas personas, cuando recibían información y educación financiera, además, se planteó que existe correlación significativa en la planeación financiera, de manera particular en planes de ahorro e inversión. Los estudios fueron realizados en países desarrollados, enfocados a individuos de escasos recursos. Para países en desarrollo, según las investigaciones realizadas por los entes gubernamentales (Lusardi y Mitchell 2016), se encontró que estos programas son indispensables, porque mejoran las habilidades para optimizar los recursos en beneficio propio.

En contraste con estos estudios realizados durante el proyecto "Insólito", de la Fundación Junior Achievement Colombia, se encuentran trabajos sin correlación entre la educación financiera y el comportamiento de los individuos; en el 2001 realizó el estudio en 79 escuelas, donde analizaron el comportamiento entre los estudiantes que asistieron y que no lo hicieron al curso de manejo de finanzas personales, y encontraron muy pocas diferencias, por eso llegaron a la conclusión de cuestionar la efectividad de los cursos.

\section{¿Cuáles son las Características de la capacitación?}

Con el fin de llegar a más personas, usando el voz a voz, los cursos deben ser desarrollados en lenguaje sencillo y claro, evitando los términos técnicos, esto hace que su efectividad aumente y los resultados sean más palpables. En Estados Unidos, según los estudios realizados por la Universidad de Columbia (1997), la implementación de la Educación Financiera en las escuelas públicas de manera obligatoria fue la causa del aumento de la sanidad financiera de los estudiantes en su etapa de adultos. Estos cursos son más beneficiosos cuando se trata de información general que ayuda a los estudiantes en la toma de decisiones de manera acertada.

Según este estudio, se deben tener en cuenta cuatro (4) enfoques:

1. Tomar decisiones informadas, lo que permitirá cumplir sus metas financieras.

2. Tener en cuenta el comportamiento de la economía (inflación - deflación; oferta - demanda).

3. Finanzas personales: Presupuesto familiar, manejo del crédito y del dinero. Inversiones.

4. Los derechos y deberes que se tiene como consumidor financiero.

El éxito de la educación financiera está también sujeta al canal por el que se difunda; por ejemplo, las finanzas del hogar pueden ser divulgadas por medio de talleres a la comunidad, reunión con líderes comunitarios (multiplicadores de información), también por medios de comunicación masiva.

Al abordar al público indicado, con la información indicada, por medio de talleres, videos y congresos, se garantiza el éxito y la optimización de los recursos, se pueden encontrar segmentos interesantes en lugares de trabajo, 
instituciones educativas, iglesias, cooperativas. Para llegar masivamente, se pueden usar medios como el Internet, la radio y la televisión.

Los estudios de CEMLA se realizaron para establecer cuál sería el canal más eficiente para brindar esta clase de información, por medio de encuestas a consumidores activos en los Estados Unidos en los meses de noviembre y diciembre del año 2001; se estableció que las decisiones son tomadas, en primera instancia, de acuerdo con la experiencia personal e individual, luego acude a familiares y amigos, por último, tienen en cuenta los medios de comunicación masiva.

En India, los canales masivos como la radio, fueron los usados por la Organización CARE (Burnas y Dalal, 2010), igual hizo el Ministerio de Planeación Financiera y Económica de Ghana (Gyabaah, 2009).

La incidencia que tienen los talleres emitidos usando medios masivos es que, por vías diferentes generan cambios en los individuos; la primera vía es la corrección en la percepción de la gente, o la generación de conocimiento por tener acceso a nueva información; y la segunda vía, es la capacidad que tienen los medios masivos de influenciar inconscientemente a las personas. En contraste, los estudios de CEMLA arrojaron que los medios escritos eran los más efectivos para la educación familiar.

\section{La Evolución de la Educación Financiera En Seguros}

Los países que se han distinguido por tener iniciativas innovadoras y atractivas en educación en seguros son: Kenia, Suráfrica, México, India y Brasil (Mejía Delgado 2012). En estos países, gracias a las agremiaciones, se han logrado definir contenidos y realizar difusión de estos por medio de talleres, Internet, concursos, ferias, Campañas en radio y televisión.

En México, la Asociación Mexicana de Instituciones de Seguros (AMIS) ${ }^{1}$ realiza desde el año 2008 seminarios, cursos presenciales en más de 500 colegios, en grados de educación primaria, videos y concursos en línea en su página Web, donde además dispone información y consejos prácticos sobre diferentes temas de seguros.

De otra parte, en Kenia, la Asociación de Seguros de Kenia (AKI) genera talleres y diferentes alternativas de educación en seguros que son difundidas por radio y televisión. En Suráfrica, la Asociación Surafricana de Seguros (SAIA) financia las estrategias de educación en seguros que diseña con el $0.2 \%$ de las utilidades de las empresas que son afiliadas. En estas campañas se han promovido principios y buenas prácticas en el desarrollo del programa, la emisión de radionovelas en lenguas nativas es un buen ejemplo.

\section{Educación Financiera En Colombia... Gestación y Avances}

La regulación de este tema se desarrolló en nuestro país con la Ley 1328 del 5 de julio de 2009, que buscó promover la participación de las entidades vigiladas por la Superintendencia Financiera, en la construcción de la protección al Consumidor Financiero y de diferentes alternativas para generar educación financiera. Dicha ley, en su artículo séptimo, obliga a la creación de campañas de educación por parte de estas entidades y establece que la Educación Financiera es uno de los principios que rigen las relaciones entre los consumidores financieros y las entidades vigiladas. Los consumidores financieros tienen el derecho de recibir información adecuada de los productos y servicios que puede recibir, sus costos y los diversos mecanismos de protección con que cuenta para la defensa de sus derechos (Documento Conpes 3424, 2006).

1 La Asociación Mexicana de Instituciones de Seguros (AMIS), es el organismo encargad de regular, investigar e implementar políticas sobre todos los tipos de seguros que se ofrecen en México, haciendo cambios, regulaciones y pactos en favor de los clientes y las aseguradoras. Página web: https://bit.ly/3nYhNrX 
Para conseguir alcanzar esta meta, la Superintendencia Financiera, en el año 2010, reglamentó el Sistema de Atención al Consumidor Financiero por medio de la Educación Financiera, mediante la emisión de la Circular Externa 015.

Uno de los mayores logros en este tema fue con el artículo 145 de la Ley 1450 de 2011, con el cual se emitió el Plan Nacional de Desarrollo 2010 - 2014, en donde se indicó que el Ministerio de Educación Nacional iniciaría el desarrollo de programas que incentivarían la Educación Económica y Financiera en el país. Facilitando la labor, hasta ese momento realizado por las entidades vigiladas por la Superintendencia Financiera; en donde conjuntamente establecieran una estrategia nacional de amplio alcance.

Iniciando el Siglo XXI, el Banco de la República realizó publicación de material impreso, en el que mediante lenguaje sencillo se pretendía llegar a los niños, contando con la tutoría de sus maestros, en sus aulas escolares con temas como economía, funciones del Banco, política monetaria. Durante los primeros años el Banco de la República distribuyó cerca de 45.000 ejemplares de cartillas con títulos como: ¿Qué es el dinero?, ¿Qué son los bancos?, el fantasma de la inflación, entre otros. En este mismo contexto, el Banco desarrolló 4 guías para ser difundidas en los Proyectos Educativos Institucionales de los colegios, en donde se abordan ejes temáticos como: El Sistema Económico, Dinero y política monetaria, Papel del Gobierno y política fiscal y Globalización Económica (FASECOLDA 2016). Así mismo, desde el 2006, el Banco de la República realiza charlas y conferencias sobre finanzas personales, visitas guiadas a la planta de distribución de billetes y concursos para los estudiantes en temas de economía.

A finales del año 2007 surge por parte del sector asegurador colombiano la inquietud por desarrollar un programa de educación financiera en riesgos y seguros; esto como resultado del análisis de distintas encuestas de la calidad de vida, y en especial de las personas de menores ingresos, las cuales se encuentran más expuestos a riesgos tales como: desastres naturales, desempleo, enfermedades y muerte. Cuando estos eventos ocurren son cubiertos con rubros que se consiguen gracias a la disminución del consumo de alimentos, el endeudamiento informal, la deserción escolar y el gasto de ahorros que se habían realizado para la compra de vivienda o el estudio de los hijos.

Algunos grupos focales realizados por Fasecolda, dieron como resultado que para los hogares menos favorecidos los seguros son un lujo y algo que no entienden. Estos resultados coinciden con los encontrados en la encuesta cuantitativa realizada por la misma institución, cuyo grupo objetivo fueron 550 hogares de los estratos uno, dos y tres en la ciudad de Bogotá. Se evidenció la falta de entendimiento de los conceptos básicos y del funcionamiento de los seguros, al igual de las diferencias y ventajas que tienen frente a los ahorros. De acuerdo con esto, se confunden los conceptos de seguros y ahorros.

El riesgo citado más frecuentemente referido fue el desempleo, junto a la muerte de alguno de los jefes del hogar; en estos casos un crédito mitiga los riesgos dando acceso a dinero que permite solventar las necesidades básicas de los hogares en el corto plazo, lo que a mediano y largo plazo genera incertidumbre, por cuanto la materialización de alguno de los riesgos afecta la capacidad de afrontar el pago de las deudas adquiridas (Informe Ejecutivo Fasecolda, 2016).

De las encuestas realizadas a finales del 2007, para analizar la calidad de vida de las personas con menores ingresos, el $40 \%$ de las personas encuestadas ahorra pensando en hacerle frente con dichos ahorros a emergencias costosas, como el desempleo o una enfermedad grave. Frente a esta última, un $20.5 \%$ asegura que usaría sus ahorros programados, mientras que el $27.3 \%$ no tiene interés en asegurarse; cuando se les consultó sobre conocimientos en seguros, este resultó muy vago. El 5.2\% de los encuestados no tiene seguros porque no los entiende, lo que hizo que en el año 2008 el sector asegurador desarrollara un programa de capacitación financiera con énfasis en seguros. 
El programa desarrollado por las aseguradoras pretendía brindar herramientas para aumentar la conciencia sobre los riesgos y la manera como deben mejorar sus conductas frente a ellos, con el ánimo de que se tomen mejores decisiones y más informadas, sobre el manejo de los riesgos y la compra de seguros.

Los objetivos de este programa fueron:

a) Reflexionar sobre los riesgos y sus costos inmediatos y en el mediano plazo.

b) Reconocer las medidas de respuesta ante las emergencias y su efectividad a corto, mediano y largo plazo.

c) Identificar las estrategias financieras para manejar los riesgos, tales como el ahorro, el crédito y los seguros.

d) Desarrollar habilidades para evaluar la efectividad de dichas herramientas financieras, para superar situaciones de riesgo.

e) Reconocer el papel de los seguros en la protección de los riesgos y su funcionamiento.

f) Tener herramientas que permitan comparar los diferentes tipos de pólizas de seguros y comunicarse con un asesor.

g) Contar con la habilidad de comprender las condiciones incluidas en una póliza de seguros.

h) Reconocer los deberes y derechos que tienen como consumidores financieros y los mecanismos que dispone la ley para su defensa (FASECOLDA 2016).

Como conclusión, se estableció que la población objetivo estaría compuesta por población de todos los estratos, pero haciendo énfasis en los más pobres, que desconozcan de seguros y tengan interés en aprender del tema, información que les permita tomar decisiones informadas sobre la administración de riesgos y para la protección de sus derechos como consumidores financieros. Para el desarrollo de este programa, Fasecolda logró firmar un Convenio Marco de Cooperación con el SENA, quien disponía de sus docentes y asumía la remuneración de estos; por su parte, Fasecolda era responsable de conseguir a los interesados en recibir la capacitación, de la logística y el material. Las entidades beneficiarias, como las aseguradoras y/o instituciones microfinancieras, recibirían la capacitación sin ningún costo y serían responsables de hacer la convocatoria de los asistentes, así como de ubicar y disponer del aula en el cual se realizará la capacitación.

Entre 2009 y 2010 se beneficiaron 64 instructores del SENA, estos capacitaron a 5953 alumnos, el 89\% beneficiarios del subsidio al desempleo de la Caja de Compensación Familiar Compensar, y el 11\% entre clientes de compañías de seguros y otros, la mayoría de estos en Bogotá. Se dictaron 235 cursos, con una asistencia promedio de 25 alumnos y 2820 horas dictadas. La tasa de aprobación del curso fue del $75 \%$. El $60 \%$ de los alumnos fueron mujeres y el $59 \%$ personas entre los 18 y 39 años.

Se pensó en contar con una herramienta adicional que permitiera un mayor alcance, por lo que en el 2010 Fasecolda adelantó un programa piloto de radio que tuvo una audiencia estimada de 17000 oyentes. La emisora escogida fue Fiesta 1340 AM, de la cadena RCN. Fasecolda trabajó en los contenidos, los cuales tienen como cimiento los talleres de formación. Para el desarrollo de los contenidos se tuvo en cuenta recomendaciones de autores como Tremblay (1974) y Grover y Miller (1976) y entidades como Radio Farm, quienes coinciden que es importante adaptar el lenguaje para captar la atención del público objetivo, definir con claridad el objetivo del mensaje y avanzar en cada emisión hacia el objetivo deseado. Los contenidos del programa se desarrollaron en los bloques que se describen en la Tabla 1.

\begin{tabular}{|c|c|c|c|c|c|}
\hline No. Semanas/bloque & Miércoles & Jueves & Viernes & Lunes & Martes \\
\hline & & & & & \\
\hline $\begin{array}{l}\text { ¿Qué riesgos } \\
\text { reconoce y cuáles le } \\
\text { han pasado a usted? } \\
\text { ¿Cómo afectaron su } \\
\text { bolsillo? }\end{array}$ & 1. Introducción & 2. Muerte & 3. Enfermedad e invalidez & 4. Desempleo & $\begin{array}{l}\text { 5. Accidentes } \\
\text { personales y de } \\
\text { tránsito }\end{array}$ \\
\hline
\end{tabular}




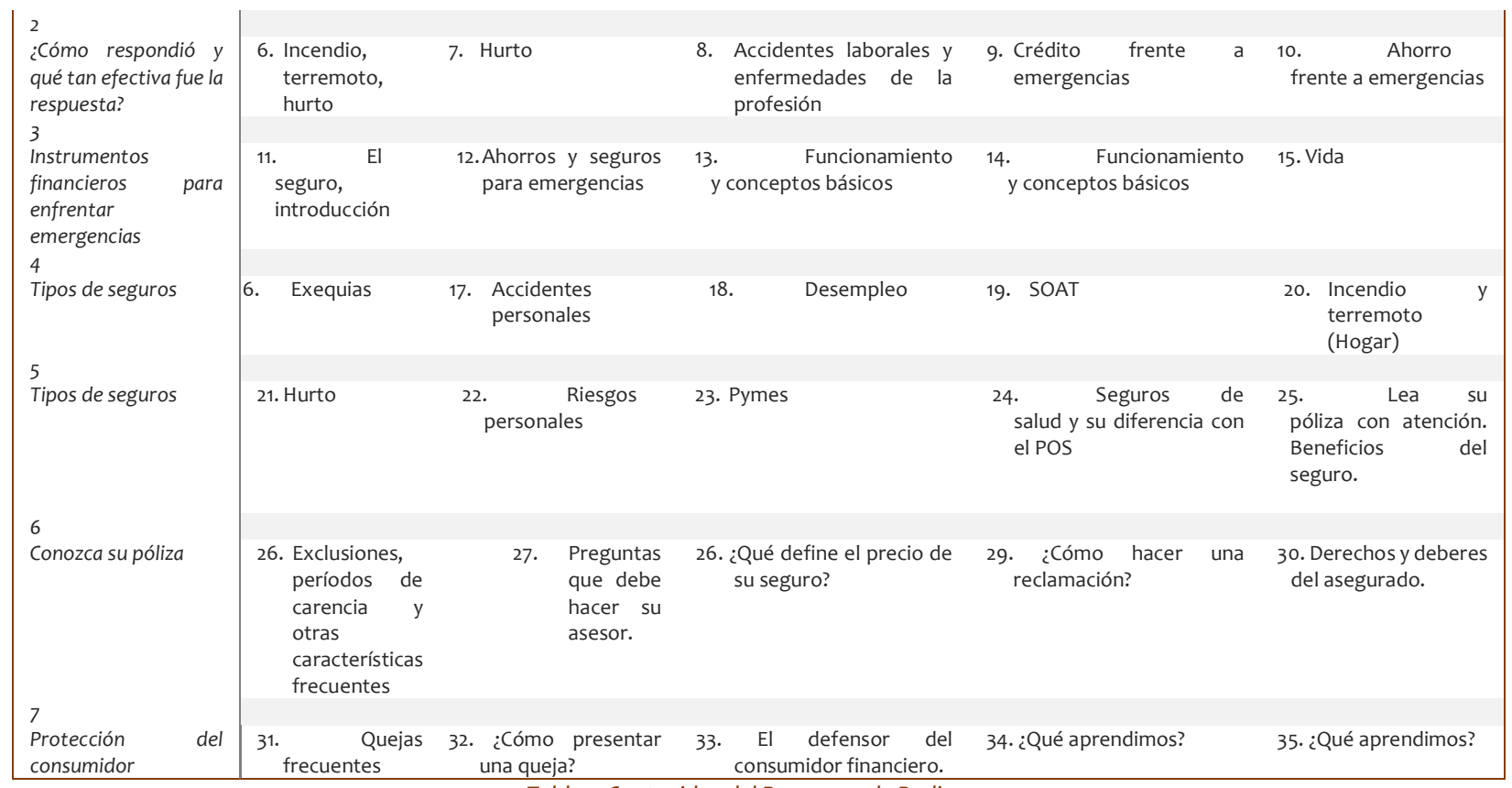

Tabla 1. Contenidos del Programa de Radio

En los talleres presenciales, la capacitación se basó en ejercicios prácticos, de clasificación, asociación y discusión; en la radio, por consejo de los expertos, se realizó la capacitación por medio de diferentes temáticas como radionovelas, notas de interés, entrevistas con expertos, entre otros, como se observa en la Tabla 2, con el fin de hacer un programa dinámico, dando espacio a la reflexión y que el público tuviera acceso a información veraz y oportuna de fácil comprensión.

\begin{tabular}{|c|c|c|c|}
\hline No./Sección & Minutos & Segundos & Observaciones \\
\hline 1. Presentación pregrabada & & 15 & Canción que identifica el programa \\
\hline 2. Bienvenida & 3 & & Repaso general, episodio anterior y estructura del día \\
\hline $\begin{array}{l}\text { 3. A usted también le puede } \\
\text { pasar }\end{array}$ & 3 & & Tres entrevistas breves \\
\hline 4. Información comercial & & 15 & Comercial \\
\hline 5. Hablemos con el experto & 6 & & Experto tema macro \\
\hline 6. Canción 1 & 3 & 30 & Relacionado con el tema del día \\
\hline 7. Notas seguras & & 30 & Notas curiosas ¿Sabía usted que...? \\
\hline 8. Radionovela & 3 & & Sobre el tema del día \\
\hline 9. Aprenda, participe y gane & 2 & & Concurso de la semana \\
\hline 10. Casos y cosas & 3 & & Tres entrevistas breves \\
\hline 11. Canción 2 & 3 & 30 & Relacionada con el tema del día \\
\hline 12. Información comercial & & 15 & Comercial \\
\hline 13. Hablemos con el experto 2 & 6 & & Experto micro, psicólogo, antropólogo, académico \\
\hline 14. Viva seguro, viva tranquilo & 1 & 30 & Recomendaciones sobre el tema del día \\
\hline $\begin{array}{l}\text { 15. ¿Está usted seguro? } \\
\text { Escríbanos. }\end{array}$ & & 30 & Contactos \\
\hline 16. Canción 3 & 3 & 30 & Relacionados con el tema del día \\
\hline 17. Conclusión y despedida & 1 & 30 & Conclusiones: presentación del siguiente programa \\
\hline 18. Despedida pregrabada & & 15 & Canción que identifica el programa \\
\hline & 41 & 30 & \\
\hline
\end{tabular}

Fasecolda, con el fin de entregar a los alumnos material escrito, diseñó cartillas que les permitieran reforzar los conocimientos adquiridos. La primera edición de esta cartilla fue publicada en el año 2009 y distribuida a casi 
2,000 personas. En 2010 se desarrolló la segunda edición de esta cartilla, la cual fue distribuida a casi 3000 personas asistentes a la feria Colombia Responsable y a otros eventos.

Desde el 01 de enero de 2011 entró en vigor la ley 1328 de 2009, que reglamentó el Sistema de Atención al Consumidor Financiero, y en lo que hace referencia a los programas de Educación Financiera, las entidades que deben realizarlas y que deben ser vigiladas por la Superintendencia Financiera. En octubre de 2010 se creó formalmente el Comité de Educación Financiera de Fasecolda. Este Comité está conformado por un delegado de cada una de las Compañías afiliadas al gremio, el Vicepresidente Ejecutivo de Fasecolda, un delegado de los Defensores del Consumidor Financiero de las Compañías de Seguros, el Director de Comunicaciones de la Agremiación, el Director del Instituto Nacional de Seguros y el Director de la Fundación de Aseguradores Colombianos (Fundaseg), y de la Fundación Educativa Centralseguros (FEC). Estas entidades son adscritas al medio y su función es la financiación de la educación financiera. Por unanimidad en la votación de la Junta Directiva del Gremio, compuesta por los presidentes de las compañías de seguros, se adelantaría la educación financiera como iniciativa de carácter social, para lo cual se creó la Dirección de Responsabilidad Social de Fasecolda, con lo cual se pretendía garantizar la transparencia con los grupos de interés como los clientes y la sociedad.

Para el desarrollo de esta estrategia, se dispuso una inversión cercana a los $\$ 1085$ millones de pesos, el 24\% de los cuales fueron aportados por las compañías de seguros, los recursos que estas dispongan para: difusión de radio, material impreso, no se incluyeron en el monto antes señalado.

Con el fin de tener un mayor cubrimiento, el sector asegurador lanzó en cada una de sus páginas web todo lo concerniente a Educación Financiera. Allí su cliente puede encontrar la explicación de cada tipo de seguro y videos explicativos de cada ramo.

\section{METODOLOGÍA}

Por medio de trabajo de campo se contó con la participación de jóvenes que se encontraban cursando décimo grado. Se realizaron 6 (seis) sesiones teórico-prácticas de 2 horas clase con duración de 90 minutos cada una, dictadas en el Colegio San José de Castilla, ubicado en la carrera 78 F \# 7 D - 03 Bogotá D.C. El trabajo incluyó el otorgamiento de las herramientas necesarias para que los jóvenes aprendieran nociones básicas de seguros por medio de talleres que permitiesen la co-creación de contenidos, generándoles preguntas sobre sí mismos y su realidad, con el fin de formarlos para que pudieran ver su entorno a través de un nuevo conocimiento. La intervención incluyó ejercicios prácticos y experiencias propias para desarrollar un pensamiento crítico y analítico, que les permitiera resolver problemas prácticos por su propia cuenta.

El trabajo buscó fomentar en los jóvenes la cultura de la prevención y protección frente a los riesgos, sensibilizándolos frente a la posibilidad de afrontar pérdidas y de cómo estas representan consecuencias nefastas y esfuerzos enormes para recuperar lo perdido; despertando en ellos la capacidad de ser consumidores informados y responsables para la toma de decisiones inteligentes y autónomas que garanticen su bienestar.

Las 6 (seis) sesiones se estructuraron de la siguiente manera:

Se realizó una lectura de un diario (periódico) de un tema específico; a partir de esta se realizaron diferentes preguntas orientadas a que el joven descubriera y construyera definiciones básicas en temas de protección y seguros. Se creó un personaje para cada sesión, que fuese de fácil recordación para el estudiante y le ayudara a sintetizar los conceptos adquiridos. En cada sesión se realizó una dinámica lúdica con el fin de facilitar la identificación y retención de lo aprendido. 
Primera Sesión: ¡Perdió el Oro!

La lectura se trataba de lo vivido en el Mundial de Patinaje cuando el antioqueño Elver Tinoco celebró su victoria 10 metros antes de llegar a la meta y mientras tanto el competidor coreano lo sobrepasó y ganó la medalla de oro. Con esto se da a entender que podemos perder lo que valoramos, identificar los riesgos y analizar como asumir la pérdida.

Personaje: Míster Fresco. Nada malo le pasa.

Segunda Sesión: ¡Cae vaca del cielo!

La lectura describe lo sucedido en Bahía Cupica, cuando un pescador fue sorprendido en su balsa por una vaca que cayó del cielo. Con esta lectura se dan a conocer los conceptos de frecuencia y severidad.

Personaje: Redondo Sabiondo. No cree en presentimientos, ni pálpitos, solo en los hechos... ¿Para qué preocuparse del resto?

\section{Tercera Sesión: ¡Egresos Brutos!}

La lectura hace referencia al concurso de televisión "La respuesta millonaria" donde el participante perdió el premio mayor de 100 millones de pesos con la pregunta... más sabe el diablo por viejo que por... Por medio de esta lectura se da a conocer la importancia del seguro, qué es y para qué sirve.

Personaje: Viejo Pimentel. Le ha tocado duro en la vida; cada vez que le ofrecen un seguro se le escucha decir... " $¡ Y$ Ya le he dicho que no me interesa! ¿O es que acaso la plata crece en los árboles como para malgastarla en seguros?

\section{Cuarta Sesión: ¡No hubo señal de humo!}

Esta lectura se refiere a que en una propiedad horizontal se contrató un trabajo de adecuación de las rejas, por un error en la manipulación de las herramientas se desató un incendio que afectó el depósito y varios apartamentos. Con esta lectura se identifican conceptos como responsabilidad, pérdidas comunes y masivas, prevención, coberturas.

Personaje: Perita Angustias. Ve el peligro en todo... No sale a la calle... No se mete a la piscina y prefiere no manejar... Perita conoce los riesgos, pero no los seguros.

\section{Quinta Sesión: ¡Puro teatro!}

La lectura se trata de la modalidad de robo en donde alguien simula que fue atropellado y, cuando el conductor del automóvil se baja a prestar ayuda, es víctima de un grupo de ladrones. Se explica el principio de la mutualidad; depende del interés en asegurarse y de la buena fe.

Personaje: Doña Terca. Siempre tiene la razón, nadie la hace cambiar de opinión... No hay quién la convenza de que la aseguradora le va a pagar.

\section{Sexta Sesión: Recapitulación}

Se hace un recuento de las 5 (cinco) sesiones anteriores, para identificar los conceptos que se querían mostrar en cada una y se construyen criterios con respecto al seguro, en grupo, para así confirmar que se cumplió con lo requerido. Esta sesión es muy importante porque en ella se valida y se generan las pruebas necesarias para que el curso en su totalidad haya comprendido todo lo concerniente a conceptos básicos e importancia del seguro.

El ejercicio contó con la siguiente participación (ver tabla 3): 


\begin{tabular}{|c|c|c|c|c|}
\hline Colegio & Curso & \# de voluntarios & $\begin{array}{l}\# \text { de estudiantes } \\
\text { beneficiados }\end{array}$ & $\begin{array}{l}\# \text { de estudiantes } \\
\text { valorados }(*)\end{array}$ \\
\hline \multirow{4}{*}{$\begin{array}{l}\text { San José de Castilla } \\
\text { (Jornada mañana y } \\
\text { tarde) }\end{array}$} & 1001 & 2 & 47 & 39 \\
\hline & 1002 & 2 & 43 & 41 \\
\hline & 1003 & 2 & 40 & 38 \\
\hline & 1004 & 2 & 38 & 38 \\
\hline \multicolumn{2}{|l|}{ TOTAL } & 8 & 168 & 156 \\
\hline
\end{tabular}

Tabla 3. Beneficiarios del Programa Insólito

(*) Sólo se tienen en cuenta aquellos estudiantes que realizaron la evaluación pre-programa y post-programa.

Por medio de actividades teórico-prácticas, de introspección y reflexión personal alrededor de nociones básicas de seguros, se orientó al adolescente a identificar las creencias y percepciones con respecto a los seguros, mediante estudios de caso, juegos de rol y análisis de personajes, desarrollando en ellos habilidades y competencias personales y ciudadanas: autonomía financiera, pensamiento crítico y resolución de conflictos.

Para iniciar el programa, se les aplicó una prueba a los alumnos con el fin de evidenciar sus conocimientos y expectativas en el tema (ver Figura 1).

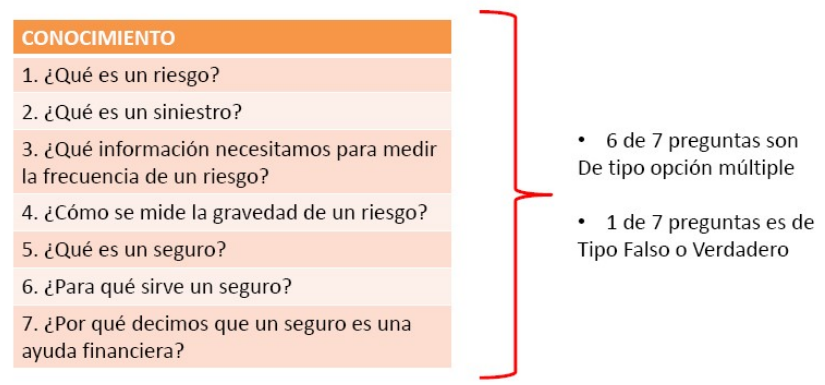

Figura 1. Herramienta de Medición Inicial

El mismo día, se aplicó el instrumento presentado en la tabla 4 que se diligenció nuevamente al final del programa. Lo que se buscaba era validar qué entendían por frecuencia y por severidad.

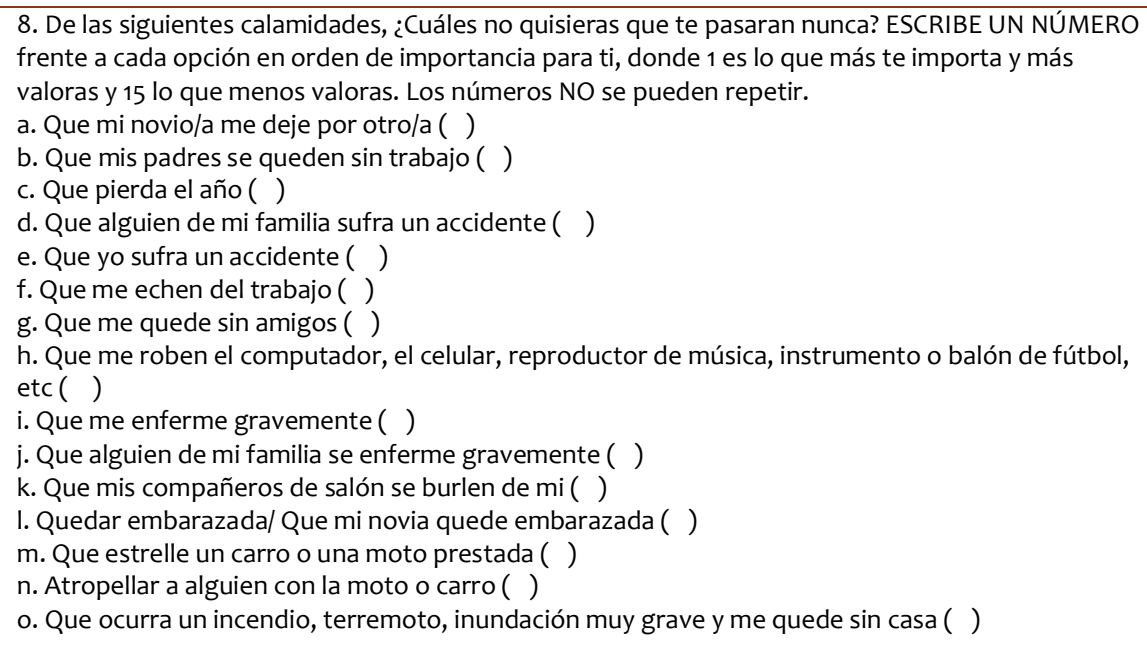




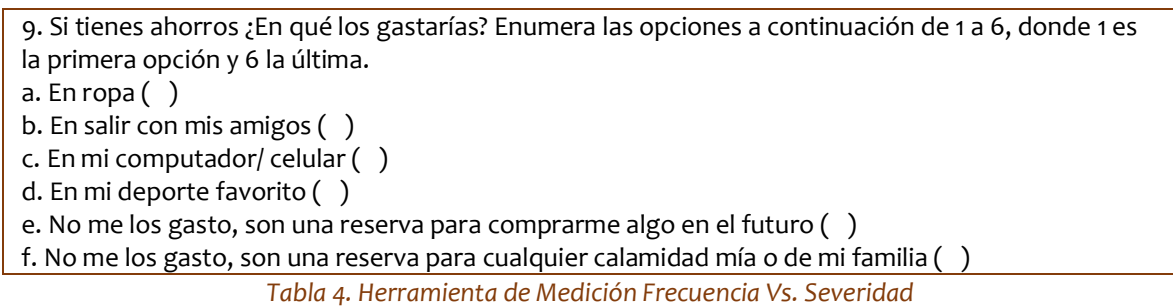

Al terminar el proyecto y tabular la información consignada en estas encuestas, el resultado fue muy bueno, optimo teniendo en cuenta que es el primer encuentro que tenemos en el colegio; se generan expectativas y retos muy grandes.

10. Al participar de este programa, tu:

\begin{tabular}{|c|l|c|c|c|c|}
\hline \multicolumn{1}{|c|}{ ENUNCIADO } & \multicolumn{2}{|c|}{ ANTES } & \multicolumn{2}{c|}{ DESPUÉS } \\
\cline { 3 - 5 } & \multicolumn{1}{|c|}{ SI } & NO & SI & NO \\
\hline 1 & Sabes qué hacer ante el riesgo & & & & \\
\hline & $\begin{array}{l}\text { Consideras que tu o lo que valoras está en condición de peligro } \\
\text { cotidianamente }\end{array}$ & & & \\
\hline 3 & Sabes qué es un seguro & & & \\
\hline 4 & Es necesario invertir dinero para proteger lo que valoras & & \\
\hline 5 & Consideras que necesitas adquirir un seguro para ti o lo que valoras & & & & \\
\hline 6 & Confías o desconfias en el funcionamiento de una compañía de seguros & & & & \\
\hline 7 & Quieres trabajar en una compañía de seguros & & & \\
\hline
\end{tabular}

Figura 2. Herramienta de Medición Expectativa Vs. Realidad.

\section{RESULTADOS}

Antes de realizar las sesiones por grupos, se aplicó una encuesta para determinar el grado de conocimientos que tenían los muchachos en temas de seguros, antes y al terminar las sesiones, lo que en las gráficas se denomina CONOCIMIENTO. Esto mismo se realizó con preguntas que pretendían determinar la EXPECTATIVA que los muchachos tenían antes y después de participar.

\section{Conocimiento}

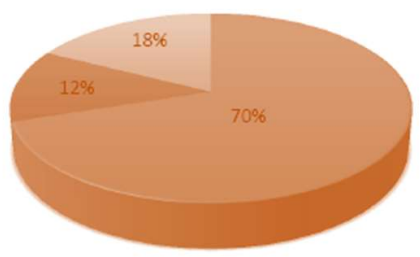

घMejoró

a No Mejoro

r.gual

Expectativa

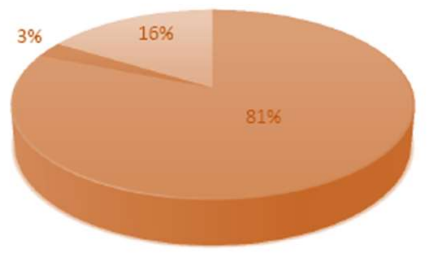

\Mejoró

- No Mejoró

⿶Igual 
MÓNICA NOVOA, HUGO GUZMÁN

Figura 5. Resultados Curso 1001

\section{CONOCIMIENTO}

घMejoró @ No mejoró @igual

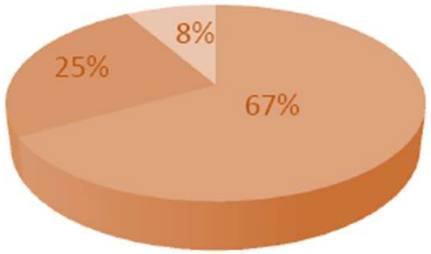

EXPECTATIVAS

a Mejoró @ No mejoró IIgual

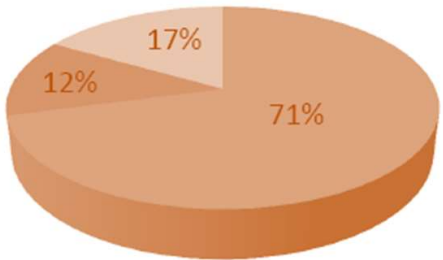

Figura 6. Resultados Curso 1002

\section{Conocimiento}

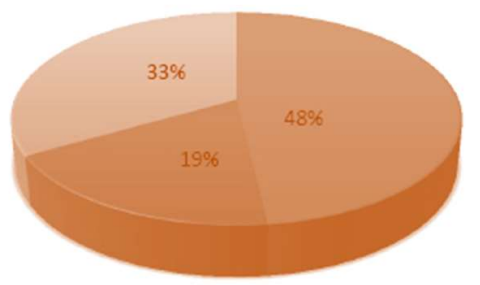

ㅂaj Mejoró

- No Mejoró

$\square$ Igual

Expectativa

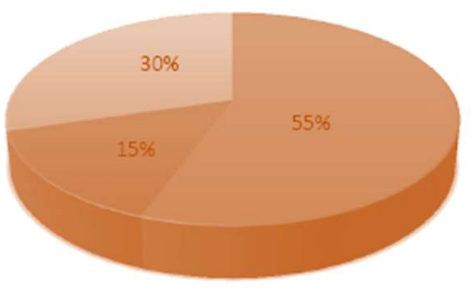

घ Mejoró

国 No Mejoró

y Igual

Figura 7. Resultados Curso 1003

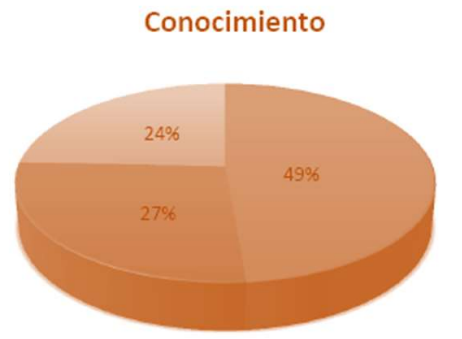

Mejoró
MNo Mejoró
IIgual

Expectativa

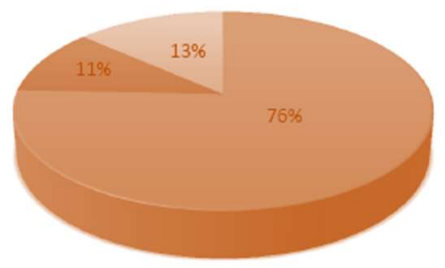

Mejoró

aNo Mejoró

IIgual

Figura 8. Resultados Curso 1004 
Realizando la validación y la tabulación de los resultados, se encuentra que en los 4 cursos fue muy positiva la respuesta y la recepción que tuvieron los muchachos a las actividades realizadas. Lo que demuestra la importancia de continuar beneficiando a más colegios con este programa.

\section{CONCLUSIÓN}

Teniendo en cuenta el recorrido realizado, se ha evidenciado que las personas que tienen acceso a la información y conocen los conceptos básicos sobre educación financiera, toman mejores decisiones en busca de proteger su patrimonio. Esto fue visto por las entidades financieras y Compañía de Seguros como una oportunidad para que las personas que manejen la información decidan adquirir sus productos basados en el análisis de sus propios riesgos. Estas entidades financieras se dieron cuenta que, según el conocimiento que pueda tener el posible cliente, este puede elegir el producto que más le convenga, que pueda pagar sin inconvenientes y sepa cómo usarlo en caso de necesitarlo.

En general, desde la educación, se deben tener en cuenta alguno aspectos, tales como:

$\checkmark \quad$ Términos usados en contratos de seguros, sus cláusulas y la presentación del producto.

$\checkmark$ Prácticas para acceder al producto y proceso de uso.

$\checkmark \quad$ Alternativas con las que el consumidor financiero cuenta para adquirir y usar el producto.

Se hace necesario la participación de nuevos agentes de cambio, empresas e instituciones que tengan conocimientos en seguros, con el fin de poder abarcar más colegios en zonas vulnerables de la ciudad y beneficiarlos con el programa.

La educación financiera puede iniciar desde el colegio y para adolescentes, pues en esta etapa, con las técnicas adecuadas, se puede incentivar el conocimiento sobre finanzas, riesgos y seguros, siendo que pare este caso se logró un muy satisfactorio 91\% de aceptación entre los muchachos beneficiados. (Encuesta Estudiantil, noviembre 2017).

\section{Referencias bibliográficas}

Ansah, Samuel Kwame, y Ernest Kissi. Technical and Vocational Education and Training in Ghana: A Tool for Skill. s.f. de enero de 2013. https://www.researchgate.net/publication/305475999_Technical_and_Vocational_Education_and_Trai ning_in_Ghana_A_Tool_for_Skill_Acquisition_and_Industrial_Development (último acceso: 30 de abril de 2021).

Banco de Desarrollo de América Latina. La educación financiera en América Latina y el Caribe. s.f. de septiembre de 2013 .

https://www.oecd.org/daf/fin/financialeducation/OECD_CAF_Financial_Education_Latin_AmericaES.pdf (último acceso: 30 de abril de 2021).

Departamento Nacional de Planeación (2006, mayo). Documento CONPES 3424.

FASECOLDA. Educación Financiera. 2016. https://fasecolda.com/fasecolda/educacion-financiera/ (último acceso: 14 de septiembre de 2017).

Figueroa, Domingo, Juan Otero, Maribel Ortiz, y Juan Rivera. Importancia de la Educación Financiera para una Decisión Confiada de Retiro. s.f. Importancia de la Educación Financiera para una Decisión (último acceso: 20 de noviembre de 2017). 
MÓNICA NOVOA, HUGO GUZMÁN

Lusardi, Annamaria, y Olivia S. Mitchell. La importancia económica de la alfabetización financera: teorías y pruebas. s.f. de diciembre de 2016. https://www.cemla.org/PDF/boletin/PUB_BOL_LXII-04-01.pdf (último acceso: 6 de febrero de 2021).

Mejía Delgado, Hernán. Seguros y Finanzas para la familia. Bogotá: Ecoe Ediciones, 2012. 